\title{
Neurofeedback and Motor Imagery are as good as physical training on Dart Throwing
}

Maryam Salehi ${ }^{1}$, Wesley Pyke ${ }^{2}$, Hasan Mohammadzadeh ${ }^{1}$, Mohammad Ali Nazari ${ }^{3}$, AmirHomayoun Javadi ${ }^{2,4,5}$ *

${ }^{1}$ School of Sports and Exercise Sciences, Urmia University, Urmia, Iran

${ }^{2}$ School of Psychology, University of Kent, Canterbury, UK

${ }^{3}$ Division of Cognitive Neuroscience, University of Tabriz, Tabriz, Iran

${ }^{4}$ Institute of Behavioural Neuroscience, University College London, London, UK

${ }^{5}$ School of Rehabilitation, Tehran University of Medical Sciences, Tehran, Iran

\section{"Corresponding author:}

Amir-Homayoun Javadi

Address: $\quad$ School of Psychology,

Keynes College,

University of Kent,

CT2 7NP, Canterbury,

Kent, UK

Phone: $\quad+441227827770$

E-Mail: $\quad$ a.h.javadi@gmail.com 


\section{Abstract}

Objectives This experiment assessed the effectiveness of three interventions; neurofeedback, motor imagery and physical training, on their ability to improve visuomotor accuracy, measured by scores achieved on a dart throwing task.

Methods Thirty-two female participants, all with no experience in dart throwing, took part and were assigned to either one of the three experimental conditions, or a control group (eight per condition). Training sessions for neurofeedback (NFB) involved participants encouraging both Theta rhythms and Sensorimotor rhythms (SMR). For Motor Imagery (MI), internal ( $1^{\text {st }}$ person) imagery was used, where participants had to imagine throwing a dart and for physical training (PT) participants physically practised dart throwing. Measurements were recorded pre-intervention, after two weeks (6 sessions) of training (mid-intervention), after 5 weeks (15 sessions) of training (in total; Outcome Measure 1) and again two weeks after training had finished (Outcome Measure 2).

Results from Mid-intervention, Outcome Measure 1 and Outcome Measure 2 were compared to baseline scores achieved pre-intervention and showed all three interventions to significantly increase performance on dart throwing compared to the Control group.

Conclusion Our findings show that, as well as traditional physical practise, both motor imagery and neurofeedback can effectively improve performance on a fine visuomotor task.

Keywords: mental imagery; motor performance; procedural memory; motor learning; visuomotor accuracy 


\section{Introduction}

Eye-hand coordination is a vital element of daily life. It allows us to respond with precise movement to visual objects at an extremely fast rate. Accuracy and ability of visuomotor coordination changes throughout the lifespan, developing throughout childhood, from infancy to adolescence (Caetano, 1995) and decreasing in older age (Lee, Kwon, Son, Nam, \& Kim, 2013). Visuomotor accuracy can be influenced further, however, depending on various factors. For example, through practise, one can become adept at a certain sport or ability related to a daily routine. This increase of skill can be attributed to long-term potentiation (LTP), a strengthening of synapses, in this instance, in the brain regions responsible for visuomotor memory (Ma et al., 2010; Packard \& Knowlton, 2002). In this study we investigated three methods that have been shown to increase performance in sports, albeit through different processes: neurofeedback, motor imagery, and physical training.

It is well known that by physically practising a task, performance improvements are inevitable. There are however other methods shown to be effective at increasing performance on visuomotor tasks. One such method is Neurofeedback (NFB). NFB provides individuals with access to current brainwave/cortical activity through the use of visual or auditory depictions provided by electroencephalography (EEG). The mechanisms of NFB training are thought to work through the use of operant conditioning (Hammond, 2011; Vernon et al., 2003), where regulation and suppression of unwanted oscillatory brainwaves allow for greater focus on tasks at hand (Vernon, 2005). Whilst improvements are initially short term, through continuous training sessions, longterm improvements have been found (ref). It has many clinical applications, including treatment for ADHD (Baumeister et al., 2016; Jahani, Pishyareh, Haghgoo, Hosseini, \& Ghadamgahi Sani, 2016), anxiety, depression, alcoholism (Peniston \& Kulkosky, 1989; Saxby \& Peniston, 1995), post-traumatic stress disorder (Peniston \& Kulkosky, 1991), autism and many more (for a review, see Hammond, 2011).

There are many theory-driven protocols of NFB, developed for a variety of needs. One protocol, Alpha/Theta, requires the participant to encourage Theta $(4-7 \mathrm{~Hz})$ rhythms over Alpha $(8-12 \mathrm{~Hz})$ (Orndorff-Plunkett, Singh, Aragón, \& Pineda, 2017). This method of NFB training was first used in a clinical setting by Eugene Peniston, who went on to publish a series of papers highlighting its usefulness for alcoholics, substance addicts and post-traumatic stress disorder (PTSD) sufferers, 
in comparison to traditional pharmacological methods (Peniston \& Kulkosky, 1989, 1991; Saxby \& Peniston, 1995). Alpha/Theta training has also yielded positive results in non-clinical settings with increases in performance found in both sports (Mirifar, Beckmann, \& Ehrlenspiel, 2017) and cognitive domains (Gruzelier, 2009).

Other protocols of NFB have been developed and implemented throughout research with the focus shifted towards performance enhancement, specifically in sport. Theta/Sensorimotor Rhythm (12$15 \mathrm{~Hz}$; SMR) protocol attempts to teach the subject to promote high frequencies of SMR, which are associated with increased attention (Kober et al., 2015), as well as increased performance in sports requiring fine motor accuracy (Cheng et al., 2015; Cheng et al., 2017). Vernon and colleagues found Theta/SMR NFB training to significantly increase performance on working memory tasks (Vernon et al., 2003). When comparing the effectiveness of both Alpha/Theta and Theta/SMR protocols, an increase in performance of ophthalmic microsurgeons was found for both, independent of each other, compared to a Control group (Tomas Ros et al., 2009).

Another method that has been previously used to improve performance in a wide range of tasks is mental imagery. Widely used amongst athletes, mental imagery can be defined as the creation or re-creation of an experience or action within the mind without direct external stimuli (Morris, Spittle, \& Watt, 2005). It has been shown that when performing mental imagery of a visual task, the same areas of the primary visual cortex $(\mathrm{V} 1, \mathrm{~V} 2)$ are activated as would be in actual visual perception (Pearson, Naselaris, Holmes, \& Kosslyn, 2015).

The use of mental imagery in sport and procedural learning can be referred to as Motor Imagery (MI). MI has been shown to be as effective as physical training (PT) for performance enhancement of a task (Haghkhah, Sohrabi, Torbati, \& Hajatmand, 2014; Kornspan, Overby, \& Lerner, 2004). When used in conjunction with PT, MI has been shown to increase performance to a greater degree than PT alone (Vaezmousavi \& Rostami, 2009). Three types of goal-directed MI have been suggested in the literature, internal ( $1^{\text {st }}$ person), external ( $3^{\text {rd }}$ person) and kinaesthetic (feelings and sensations associated with movement). Yu et al., (2016) showed when participants used internal imagery, performance was increased for a wide range of sports, whereas when external imagery was practised, increase in performance was only seen for open sports and team games. It has also been shown that whilst external imagery may allow for particular tasks to be completed faster, internal imagery can provide greater accuracy (White \& Hardy, 1995). 
Using three independent conditions and a Control group, the present study attempts to highlight the most effective method of training (NFB; MI; PT) to increase performance on a fine visuomotor task, in this instance, dart throwing. For the MI condition, internal MI was used as this has been shown to be most effective for this particular task (White \& Hardy, 1995; Yu et al., 2016). For the NFB condition, both alpha/theta and theta/SMR protocols was used, as both have been shown to improve performance on slow, self-paced tasks. By also including a two-week retention interval, the three methods can be assessed on their ability to induce LTP and facilitate long-term motor memory changes. It was hypothesised that in all three experimental conditions (NF; MI; PT), performance increases will be found following each respective training intervention (Preintervention to Outcome Measure 1 and 2). It is also hypothesised that each experimental condition will increase in performance compared to the Control group.

\section{Methods}

\section{Participants}

Thirty-two female participants (age range 21-26, mean[SD] $=22.500[1.066]$ ) took part in this study. They were randomly assigned to one of the four experimental conditions (Neurofeedback/Motor Imagery/Physical Training/Control). All participants were naïve to the purpose of the study and none of them were familiar with neurofeedback training, motor imagery nor dart throwing. They were right-handed without any history of neurological disorders with normal or corrected to normal vision. They gave written informed consent. The study was approved by the local ethics committee in the School of Sports and Exercise Sciences, Urmia University.

\section{Procedure}

Participants took part in four testing and 15 training sessions over six weeks. See Figure 1 for the procedure. In the first session, participants were familiarised with principles of dart throwing such as holding, aiming, throwing the dart, and scoring. Then, participants were asked to throw darts for three practice trials. Performance was measured via 10 attempts of attempts throwing darts and scores were recorded. Following the pre-intervention testing session, participants took part in five 
weeks of training based on their allocated group, please see below for condition. Each week consisted of three training sessions. Two weeks in the training, participants took part in a testing session to assess their mid-intervention performance. Finally, participants took part in two more testing sessions to measure their performance immediately (Outcome Measure 1) and one week (Outcome Measure 2) following training. Sessions Pre- and Mid-intervention and Outcome Measure 1 took part on a separate day than the training sessions.

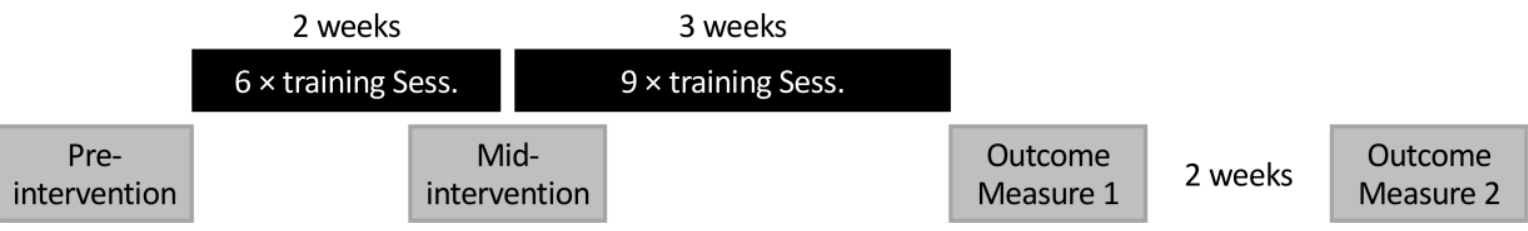

Figure 1. Procedure of the study

\section{Dartboard}

In this study, a typical circular dart board compressed with paper was used with a diameter of 453 $\mathrm{mm}$ and thickness of 12 . The dartboard was hung on the wall in the laboratory so that the centre of the dartboard was at a height of $1.72 \mathrm{~m}$ from the ground. A line was traced on the ground at a distance of $2.20 \mathrm{~m}$ from the dart board and the subjects would be behind the line when throwing the darts. Five metal-arrow darts weighing 25 grams and $15 \mathrm{~cm}$ long were used.

\section{Intervention Methods}

Neurofeedback (NFB) - FlexComp Infiniti 10 channel EEG system (Thought Technology Ltd., Canada) was used for EEG acquisition. Electrodes were placed on C3 and Pz based on 10-20 international EEG electrode placement system. Oscillatory brain activity was displayed via Biograph Infiniti (Thought Technology Ltd., Canada) in the form of coloured bars on the computer screen. Two protocols were used: Alpha/Theta and Theta/Sensorimotor Rhythm (SMR). The training protocol Alpha/Theta included recording activity in the alpha and theta frequency bands while the participant had their eyes closed. Participants were asked to relax and listen to pleasant sounds like coastal waves breaking softly. This protocol has been shown to boost brain activity in alpha and theta. Alpha/Theta training is an appropriate training pattern by which one attempts to increase theta band activity level compared to alpha. It has been shown that this combination leads 
to higher creativity and well-being (Gruzelier, 2009). Theta/SMR protocol runs with open eyes and includes an increase of SMR in the range of $12-15 \mathrm{~Hz}$ and a decrease of theta in the range of 4-7 Hz. Theta/SMR protocol has been shown to increase attention and concentration (Cheng, Huang, et al., 2015; Cheng, Hung, et al., 2015; Cheng et al., 2017). In each session before starting NFB training, baseline measures were recorded with eyes closed (electrode Pz for the Alpha/Theta protocol), and with eyes open (electrode C3 for the Theta/SMR protocol). The Alpha/Theta protocol lasted for 20 minutes based on the activity of the $\mathrm{Pz}$ electrode. In this protocol audio feedback was provided. Subsequently Theta/SMR protocols was administered for 10 minutes based on the activity of the $\mathrm{C} 3$ electrode. In this protocol audio and visual feedback were provided. Motor Imagery (MI) - Three types of goal-directed MI have been suggested in the literature, internal (1st person), external (3rd person) and kinaesthetic (feelings and sensations associated with movement). We used an internal imagery method as previous literature suggests it to be most effective for this particular task (White \& Hardy, 1995). Each motor imagery session lasted 30 minutes. Participants performed two blocks of 15 throwing attempts. Following each session, participants were asked to rate the quality of their imagination (Louis, Guillot, Maton, Doyon, \& Collet, 2008) on the scale of 1 (no mental imagery at all) to 6 (apparent mental imagery).

Physical Training (PT) - Each training session contained 15-minute of exercising in which participants performed 30 throws.

Control - Each session of the Control group consisted of 30 minutes of reading books.

\section{Data Analysis}

To account for inter-subject variability, performance in the Mid-intervention, Post-Intervention, Outcome Measure 1 and Outcome Measure 2 was normalised to the individual's performance in the Pre-intervention session. Due to a low number of participants and non-normal distribution of the data, Mann Whitney $U$ test was used to compare the normalised-performance of the participants in the different group of training (Neurofeedback, Motor Imagery and Physical Exercise) with the Control group. Cliff's Delta effect size is reported. 


\section{Results}

All participants followed the procedure of the study and took part in all of the sessions. The MI ratings showed that following the second intervention session participants were fully comfortable with imagining the throws (all ratings $>=5$ ).

Table 1 shows the summary of the performance of the participants in different groups and different testing sessions. Normalised data was calculated for each participant, see Figure 2.

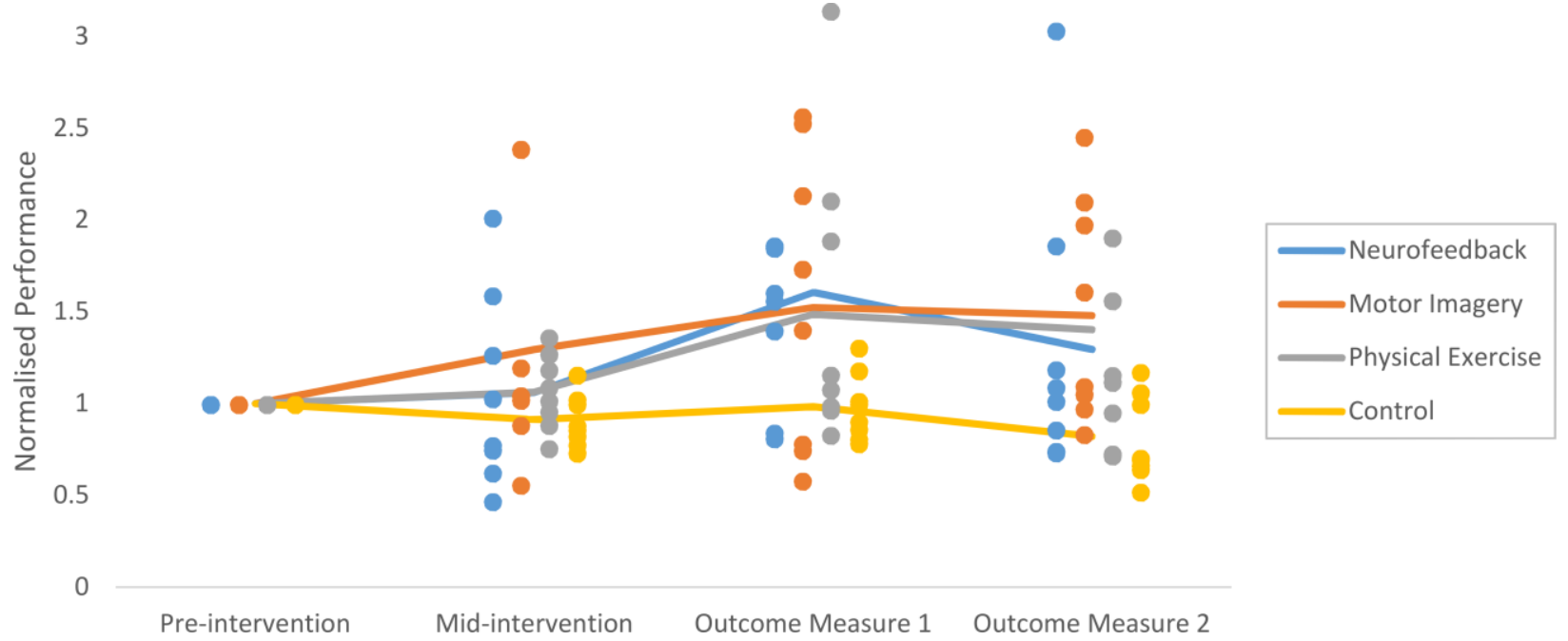

Figure 2. Normalised performance of the participants. Dots indicate individual normalised performance in each experimental group. Lines indicate the median of the values in each group and session.

Table 2 shows the summary of the Mann Whitney U tests conducted on the data. Results show that participants in all the training groups performed better in the Outcome Measure 2 compared to the Control condition.

Table 1. Summary of the median value of performance of participants

\begin{tabular}{lllll}
\hline Group & $\begin{array}{l}\text { Pre- } \\
\text { Intervention }\end{array}$ & $\begin{array}{l}\text { Mid- } \\
\text { Intervention }\end{array}$ & $\begin{array}{l}\text { Outcome } \\
\text { Measure 1 }\end{array}$ & $\begin{array}{l}\text { Outcome } \\
\text { Measure 2 }\end{array}$ \\
\hline Neurofeedback & 100 & 102 & 121.5 & 113.5 \\
Motor Imagery & 87.5 & 101.5 & 131 & 129.5 \\
Physical Exercise & 96 & 89 & 107 & 110 \\
Control & 117 & 101 & 112 & 82 \\
\hline
\end{tabular}




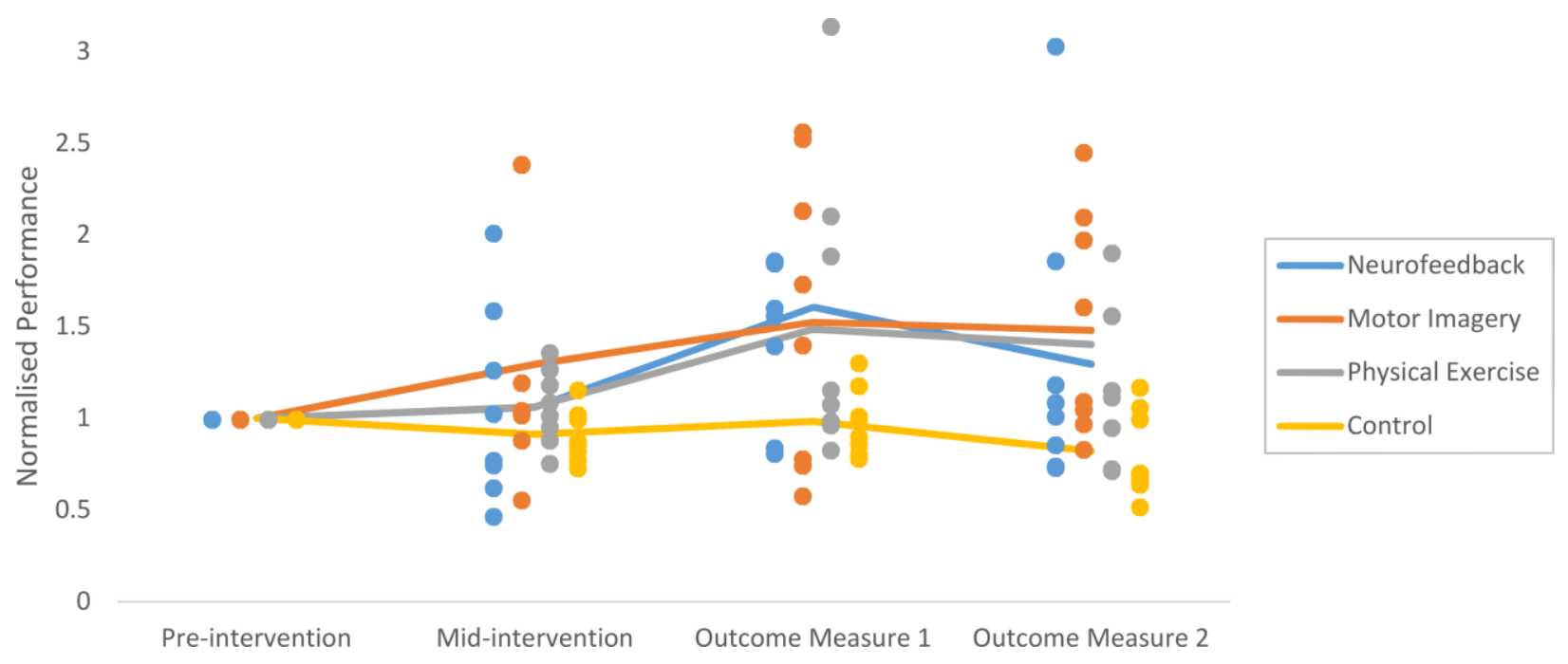

Figure 2. Normalised performance of the participants. Dots indicate individual normalised performance in each experimental group. Lines indicate the median of the values in each group and session.

Table 2. Summary of the Mann Whitney $U$ test; comparing the normalised performance of the participants in the training groups with the Control group.

\begin{tabular}{llll}
\hline Comparison & Mid-Intervention & Outcome Measure 1 & Outcome Measure 2 \\
\hline Neurofeedback vs. Control & $\mathrm{U}=30.50, p=0.875$ & $\mathrm{U}=12.50, p=0.040^{*}$ & $\mathrm{U}=12.00, p=0.036^{*}$ \\
& $\mathrm{Delta}=0.046$ & $\mathrm{Delta}=0.609$ & Delta $=0.625$ \\
\multirow{2}{*}{ Motor Imagery vs. Control } & $\mathrm{U}=15.00, p=0.073$ & $\mathrm{U}=23.50, p=0.372$ & $\mathrm{U}=9.00, p=0.016^{*}$ \\
& $\mathrm{Delta}=0.531$ & Delta $=0.265$ & Delta $=0.718$ \\
Physical Exercise vs. Control & $\mathrm{U}=16.00, p=0.092$ & $\mathrm{U}=18.00, p=0.141$ & $\mathrm{U}=11.00, p=0.027^{*}$ \\
& $\mathrm{Delta}=0.500$ & Delta $=0.437$ & Delta $=0.656$ \\
\hline
\end{tabular}

Note: normalised performance is calculated based on performance in each group divide by the performance in the pre-training phase. Cliff's Delta values are reported for effect size. ${ }^{*} p<0.05$

\section{Discussion}

As hypothesised, the results above suggest all three conditions (NFB; MI; PT) increased performance from Pre-intervention in both Outcome Measure 1 and significantly so in Outcome Measure 2 compared to the Control group. Little difference between conditions was found when comparing each of the experimental conditions, improvements from Pre-intervention to Outcome Measure 1 and 2 found across all. 
It is important to consider the mechanisms influenced by each condition in order to understand these results. MI, like PT, improved performance on dart throwing through the process of reconsolidation. By either physically practising the task, or imagining it, with the desired consequence in mind, the brain is repeatedly exposed to the same mechanisms (Newell, 1991; for a model see Adams, 1971), thus improving motor skills. Neuroimaging studies indicate that the primary motor cortex (M1) is engaged during both MI and PT of motor tasks (Kami et al., 1995) with M1 also shown to be a vital element in the consolidation process of newly learned motor skills (Muellbacher et al., 2002; Ziemann, 2004). From M1, the acquired skill follows a pathway where it will eventually reach the cerebellum (Imamizu, Kuroda, Miyauchi, Yoshioka, \& Kawato, 2003). Following successful completion of a motor action, over time, long-term potentiation (LTP) occurs in both M1 and the cerebellum (Krakauer \& Shadmehr, 2006). Failed attempted actions, however, encourage long-term depression (LTD), thus fine-tuning mechanisms which in time allows for greater accuracy and ease of execution (Hirano, 2013).

In the current study, unlike both MI and PT, the NFB training participants were exposed to, was in no way related to the dart throwing task. Instead, by gaining control over brain activity, participants were able to consciously increase or decrease oscillatory activity in certain brain regions, allowing for purposeful attentional improvements. Using EEG, Cheng et al., (2015) found skilled dart players to possess higher levels of SMR immediately before execution than non-skilled individuals, again reinforcing the involvement of SMR, particularly for dart throwing. This highlights that as dart throwing is a complex skill, and such variability was seen between the conditions, it may be useful in future to use skilled dart players, this way, scores are less likely to be attributed to chance and more to the interventions.

Whilst similar performance increases for visuomotor coordination were found in all three experimental conditions, each condition has strengths and weaknesses for practical implementation. For example, if one wanted to increase skill in a sport, it would be appropriate to use any of the three methods; although MI techniques and PT are easily accessible so may provide favourable options. In a clinical setting, however, some of the methods may not be accessible to the entire population. By highlighting that all three are as effective as one another, the appropriate method can be chosen to suit the need of the individual. An example of the suggested use of each method is shown in Table 3. 
Table 3. Suggested application of methods.

\begin{tabular}{llll}
\hline & $\begin{array}{l}\text { Physical } \\
\text { Training }\end{array}$ & Motor Imagery & Neurofeedback \\
\hline Brain damaged patients & $\times$ & $\times$ & $\times$ \\
Physically disabled & & $\times$ & $\times$ \\
Physical fitness & $\times$ & & \\
General cognitive enhancement & $\times$ & & $\times$ \\
\hline
\end{tabular}

Those suffering a brain lesion (region dependent, for example, visual object agnosia, see Behrmann, Winocur, \& Moscovitch, 1992) may be able to partake in MI for rehabilitation, but not PT. In patients suffering from a stroke affecting motor deficits, when used alongside traditional rehabilitation methods, MI was shown to aid motor rehabilitation significantly more than those methods alone (Page, Levine, Sisto, \& Johnston, 2001). Moreover, MI can be used in most instances for rehabilitation of motor skills (Jackson, Lafleur, Malouin, Richards, \& Doyon, 2001) including physical injuries in sport (Green, 1992), unless damage is present in the parietal lobes (Sirigu et al., 1996).

Another method that can be used for rehabilitation, as well as a tool to interact with external world, of both neurological diseases and physical disabilities, is brain-computer interface (BCI). BCI acquires brain signals using EEG, analyses the signals and transforms the activity into commands that are then sent to an output device (e.g., a prosthetic limb or electronic wheelchair; Shih, Krusienski, \& Wolpaw, 2012). Training to use a BCI requires some form of NFB, in order to gain control over brain waves associated with motor control (typically SMR). Hwang, Kwon and Im (2009) used a MI type NFB, displaying real-time brain activation maps during imagery to participants. They found MI improved control and performance for BCI, with those partaking in MI condition able to control SMR to a greater degree, thus increasing the effectiveness of the BCI (see also; Kondo, Saeki, Hayashi, Nakayashiki, \& Takata, 2015). Similarly, NFB alone has been shown to improve motor performance on an individual suffering from a traumatic brain injury (Wing, 2001).

Whilst there have been studies comparing the effectiveness of both Alpha/Theta and Theta/SMR independently of one another (see Ros et al., 2009; Vernon et al., 2003), to the authors' knowledge, this is the first experiment subjecting participants to more than one protocol of NFB in a single 
condition. Rationale for including both protocols was formulated on the basis that encouraging Theta frequencies in the brain is beneficial to working memory performances (Klimesch, Doppelmayr, Schimke, \& Ripper B., 1997) and increasing SMR before execution of a fine motor task, has been shown to increase attention, which in turn, improves performance (Cheng, Huang, et al., 2015; Cheng et al., 2017; Ji-Ho, Min-Jung, \& Yu-Jin, 2017).

In the context of performance in sport, NFB may extend further than just attentional and working memory processes, with research suggesting that SMR may help to facilitate early acquisition of a motor task (T. Ros, Munneke, Parkinson, \& Gruzelier, 2014) and the promotion of Theta rhythms may encourage LTP in hippocampal regions (Abrahamsson, Lalanne, Watt, \& Sjöström, 2016; Law \& Leung, 2018). This promising discovery may allow the use of NFB training to be used in the rehabilitation of injuries requiring relearning of actions or movements such as stroke, traumatic brain injury.

This study has highlighted the usefulness of neurofeedback, motor imagery and physical training in their ability to enhance visuomotor coordination based on scores achieved on a dart throwing task. Whilst we have shown the effectiveness of all three protocols in a sample of healthy students, future research should use a patient and elite samples, to assess the effectiveness of individual methods in more specific populations. With other recent promising research, as well as the results achieved in this study, the effects of these non-pharmacological methods of cognitive enhancement can stretch into multiple domains.

\section{Conflict of Interest}

Authors indicate no conflict of interest. 


\section{References}

Abrahamsson, T., Lalanne, T., Watt, A. J., \& Sjöström, P. J. (2016). Long-Term Potentiation by Theta-Burst Stimulation Using Extracellular Field Potential Recordings in Acute Hippocampal Slices. Cold Spring Harbor Protocols, 2016(6), pdb.prot091298. https://doi.org/10.1101/pdb.prot091298

Adams, J. A. (1971). A closed-loop theory of motor learning. Motor Behaviour, 3(1), 11-50.

Baumeister, S., Wolf, I., Holz, N., Boecker-Schlier, R., Adamo, N., Holtmann, M., ... Brandeis, D. (2016). Neurofeedback Training Effects on Inhibitory Brain Activation in ADHD: A Matter of Learning? Neuroscience, 378, 89-99.

https://doi.org/10.1016/j.neuroscience.2016.09.025

Behrmann, M., Winocur, G., \& Moscovitch, M. (1992). Dissociation between mental imagery and object recognition in a brain-damaged patient. Nature, 359, 636. Retrieved from https://doi.org/10.1038/359636a0

Caetano, C. (1995). Development of Visuomotor Coordination in School-Age Children: The Bimanual Coordination Test. Developmental Neuropsychology, 11(2), 181-199. https://doi.org/10.1080/87565649509540612

Cheng, M. Y., Huang, C.-J., Chang, Y.-K., Koester, D., Schack, T., \& Hung, T.-M. (2015). Sensorimotor Rhythm Neurofeedback Enhances Golf Putting Performance. Journal of Sport and Exercise Psychology, 37(6), 626-636. https://doi.org/10.1123/jsep.2015-0166

Cheng, M. Y., Hung, C. L., Huang, C. J., Chang, Y. K., Lo, L. C., Shen, C., \& Hung, T. M. (2015). Expert-novice differences in SMR activity during dart throwing. Biological Psychology, 110, 212-218. https://doi.org/10.1016/j.biopsycho.2015.08.003

Cheng, M. Y., Wang, K. P., Hung, C. L., Tu, Y. L., Huang, C. J., Koester, D., ... Hung, T. M. (2017). Higher power of sensorimotor rhythm is associated with better performance in skilled air-pistol shooters. Psychology of Sport and Exercise, 32, 47-53. https://doi.org/10.1016/j.psychsport.2017.05.007

Green, L. B. (1992). The Use of Imagery in the Rehabilitation of Injured Athletes. The Sport Psychologist, 6(4), 416-428. 
Gruzelier, J. (2009). A theory of alpha/theta neurofeedback, creative performance enhancement, long distance functional connectivity and psychological integration. Cognitive Processing, 10(1 SUPPL.), 101-109. https://doi.org/10.1007/s10339-008-0248-5

Haghkhah, A., Sohrabi, M., Torbati, H. T., \& Hajatmand, H. (2014). The Effect of Mental Imagery Focus of Attention on Performance and Learning of Children Dart Throwing Skill The Effect of Mental Imagery Focus of Attention on Performance and Learning of Children Dart Throwing Skill, (January).

Hammond, D. C. (2011). What is Neurofeedback: An Update. Journal of Neurotherapy, 15(4), 305-336. https://doi.org/10.1080/10874208.2011.623090

Hirano, T. (2013). Long-term depression and other synaptic plasticity in the cerebellum. Proceedings of the Japan Academy, Series B, 89(5), 183-195. https://doi.org/10.2183/pjab.89.183

Hwang, H. J., Kwon, K., \& Im, C. H. (2009). Neurofeedback-based motor imagery training for brain-computer interface (BCI). Journal of Neuroscience Methods, 179(1), 150-156. https://doi.org/10.1016/j.jneumeth.2009.01.015

Imamizu, H., Kuroda, T., Miyauchi, S., Yoshioka, T., \& Kawato, M. (2003). Modular organization of internal models of tools in the human cerebellum. Proceedings of the National Academy of Sciences, 100(9), 5461-5466.

Jackson, P. L., Lafleur, M. F., Malouin, F., Richards, C., \& Doyon, J. (2001). Potential role of mental practice using motor imagery in neurologic rehabilitation. Archives of Physical Medicine and Rehabilitation, 82(8), 1133-1141.

Jahani, M., Pishyareh, E., Haghgoo, H., Hosseini, S., \& Ghadamgahi Sani, S. (2016). Neurofeedback Effect on Perceptual-Motor Skills of Children with ADHD. Iranian Rehabilitation Journal, 14(1). https://doi.org/10.15412/J.IRJ.08140107

Ji-Ho, K., Min-Jung, W., \& Yu-Jin, K. (2017). The Effect of Neurofeedback Training on Shooting Performance and Cortical Activation. Journal of Korean Association of Physical Education and Sport for Girls and Women, 31(3), 153-167. Retrieved from http://www.dbpia.co.kr/Journal/ArticleDetail/NODE07245977

Kami, A., Meyer, G., Jezzard, P., Adams, M. M., Turner, R., \& Ungerleider, L. G. (1995). 
Functional MRI evidence for adult motor cortex plasticity during motor skill learning. Nature, 377(6545), 155-158.

Klimesch, W., Doppelmayr, M., Schimke, H., \& Ripper B. (1997). Theta synchronization and alpha desynchronization in a memory task. Psychophysiology, 34(2), 169-176. https://doi.org/10.1111/j.1469-8986.1997.tb02128.x

Kober, S. E., Witte, M., Stangl, M., Väljamäe, A., Neuper, C., \& Wood, G. (2015). Shutting down sensorimotor interference unblocks the networks for stimulus processing: An SMR neurofeedback training study. Clinical Neurophysiology, 126(1), 82-95. https://doi.org/10.1016/j.clinph.2014.03.031

Kondo, T., Saeki, M., Hayashi, Y., Nakayashiki, K., \& Takata, Y. (2015). Effect of instructive visual stimuli on neurofeedback training for motor imagery-based brain-computer interface. Human Movement Science, 43, 239-249. https://doi.org/10.1016/j.humov.2014.08.014

Kornspan, A. S., Overby, L. Y., \& Lerner, B. S. (2004). Analysis and Performance of Preperformance Imagery and Other Strategies on a Golf Putting Task. Journal of Mental Imagery, 28(3), 59-74. https://doi.org/10.1029/2009JC005369

Krakauer, J. W., \& Shadmehr, R. (2006). Consolidation of motor memory. Trends in Neurosciences, 29(1), 58-64. https://doi.org/10.1016/j.tins.2005.10.003

Law, C. S. H., \& Leung, L. S. (2018). Long-Term Potentiation and Excitability in the Hippocampus Are Modulated Differently by $\theta$ Rhythm. Eneuro, 5(6), ENEURO.023618.2018. https://doi.org/10.1523/ENEURO.0236-18.2018

Lee, N. K., Kwon, Y. H., Son, S. M., Nam, S. H., \& Kim, J. S. (2013). The Effects of Aging on Visuomotor Coordination and Proprioceptive Function in the Upper Limb. Journal of Physical Therapy Science, 25(5), 627-629. https://doi.org/10.1589/jpts.25.627

Louis, M., Guillot, A., Maton, S., Doyon, J., \& Collet, C. (2008). Effect of imagined movement speed on subsequent motor performance. Journal of Motor Behavior, 40(2), 117-132.

Ma, L., Wang, B., Narayana, S., Hazeltine, E., Chen, X., Robin, D. A., ... Xiong, J. (2010). Changes in regional activity are accompanied with changes in inter-regional connectivity during 4 weeks motor learning. Brain Research, 1318, 64-76. https://doi.org/10.1016/j.brainres.2009.12.073 
Mirifar, A., Beckmann, J., \& Ehrlenspiel, F. (2017). Neurofeedback as supplementary training for optimizing athletes' performance: A systematic review with implications for future research. Neuroscience and Biobehavioral Reviews, 75, 419-432. https://doi.org/10.1016/j.neubiorev.2017.02.005

Morris, T., Spittle, M., \& Watt, A. (2005). Imagery In Sport. Human Kinetics.

Muellbacher, W., Ziemann, U., Wissel, J., Dang, N., Kofler, M., Facchini, S., \& Hallett, M. (2002). Early consolidation in human primary motor cortex. Nature, 415(6872), 640-644.

Newell, K. M. (1991). Motor skill acquisition. Physical Therapy, 71(2), 123-139. https://doi.org/10.1146/annurev.ps.42.020191.001241

Orndorff-Plunkett, F., Singh, F., Aragón, O. R., \& Pineda, J. A. (2017). Assessing the effectiveness of neurofeedback training in the context of clinical and social neuroscience. Brain Sciences, 7(8), 1-22. https://doi.org/10.3390/brainsci7080095

Packard, M. G., \& Knowlton, B. J. (2002). Learning and Memory Functions of the Basal Ganglia. Annual Review of Neuroscience, 25(1), 563-593. https://doi.org/10.1146/annurev.neuro.25.112701.142937

Page, S. J., Levine, P., Sisto, S., \& Johnston, M. V. (2001). A randomized efficacy and feasibility study of imagery in acute stroke. Clinical Rehabilitation, 15(3), 233-240.

Pearson, J., Naselaris, T., Holmes, E. A., \& Kosslyn, S. M. (2015). Mental Imagery: Functional Mechanisms and Clinical Applications. Trends in Cognitive Sciences, 19(10), 590-602. https://doi.org/10.1016/j.tics.2015.08.003

Peniston, E., \& Kulkosky, P. (1989). $\alpha-\theta$ Brainwave Training and $\beta$-Endorphin Levels in Alcoholics. Alcoholism: Clinical and Experimental Research, 13(2), 271-279. https://doi.org/10.1111/j.1530-0277.1989.tb00325.x

Peniston, E., \& Kulkosky, P. (1991). Alpha-Theta Brainwave Neuro-Feedback for Vietnam Veterans with CombatRelated Post-Traumatic Stress Disorder. Medical Psychotherapy, 4, 47-60. https://doi.org/10.1021/ma0483701

Ros, T., Moseley, M. J., Bloom, P. A., Benjamin, L., Parkinson, L. A., \& Gruzelier, J. H. (2009). Optimizing microsurgical skills with EEG neurofeedback. BMC Neuroscience, 10. 
https://doi.org/10.1186/1471-2202-10-87

Ros, T., Munneke, M. A. M., Parkinson, L. A., \& Gruzelier, J. H. (2014). Neurofeedback facilitation of implicit motor learning. Biological Psychology, 95(1), 54-58. https://doi.org/10.1016/j.biopsycho.2013.04.013

Saxby, E., \& Peniston, E. G. (1995). Alpha-theta brainwave neurofeedback training: An effective treatment for male and female alcoholics with depressive symptoms. Journal of Clinical Psychology, 51(5), 685-693.

Shih, J. J., Krusienski, D. J., \& Wolpaw, J. R. (2012). Brain-computer interfaces in medicine. Mayo Clinic Proceedings, 87(3), 268-279. https://doi.org/10.1016/j.mayocp.2011.12.008

Sirigu, A., Duhamel, J.-R., Cohen, L., Pillon, B., Dubois, B., \& Agid, Y. (1996). Sirigu, A., Duhamel, J.-R., Cohen, L., Pillon, B., Dubois, B., \& Agid, Y. (1996). The Mental Representation of Hand Movements After Parietal Cortex Damage. Science, 273(5281), 1564-1568. Science, 273(5281), 1564-1568.

Vaezmousavi, S. M., \& Rostami, R. (2009). The Effects of Cognitive and Motivational Imagery on Acquisition, Retention and Transfer of the Basketball Free Throw. World Journal of Sport Sciences, 2(2), 129-135.

Vernon, D. (2005). Can neurofeedback training enhance performance? An evaluation of the evidence with implications for future research. Applied Psychophysiology Biofeedback, 30(4), 347-364. https://doi.org/10.1007/s10484-005-8421-4

Vernon, D., Egner, T., Cooper, N., Compton, T., Neilands, C., Sheri, A., \& Gruzelier, J. (2003). The effect of training distinct neurofeedback protocols on aspects of cognitive performance. International Journal of Psychophysiology, 47(1), 75-85. https://doi.org/10.1016/S01678760(02)00091-0

White, A., \& Hardy, L. (1995). Use of different imagery perspectives on the learning and performance of different motor skills. British Journal of Psychology, 86, 169-180. https://doi.org/10.1111/j.2044-8295.1995.tb02554.x

Wing, K. (2001). Effect of Neurofeedback on Motor Recovery of a Patient with Brain Injury: A Case Study and Its Implications for Stroke Rehabilitation. Topics in Stroke Rehabilitation, $8(3), 45-53$. 
Yu, Q. H., Fu, A. S. N., Kho, A., Li, J., Sun, X. H., \& Chan, C. C. H. (2016). Imagery perspective among young athletes: Differentiation between external and internal visual imagery. Journal of Sport and Health Science, 5(2), 211-218. https://doi.org/10.1016/j.jshs.2014.12.008

Ziemann, U. (2004). Learning Modifies Subsequent Induction of Long-Term Potentiation-Like and Long-Term Depression-Like Plasticity in Human Motor Cortex. Journal of Neuroscience, 24(7), 1666-1672. https://doi.org/10.1523/JNEUROSCI.5016-03.2004 